procedure and vitrectomy were planned. During vitrectomy, however, it was noted that the Ocutome tip did not reach the posterior pole. Vitrectomy was completed as far posteriorly as possible. This proved sufficient to reattach the retina when combined with scleral buckling and an air-fluid exchange. Six weeks after surgery, the axial length of both eyes was measured by A-scan ultrasonography and found to be $30.64 \mathrm{~mm}$ OD and $32.94 \mathrm{OS}$.

Comment. - When performing vitreous surgery on highly myopic eyes, the Ocutome vitrector may fall short of certain requirements. Had vitrectomy been required near the posterior pole in our patient, we believe it would not have been possible. The Ocutome is a popular choice among vitrectomy instruments and offers excellent maneuverability because of its compactness. The intraocular tip length, however, is $25 \mathrm{~mm}$, shorter than most other

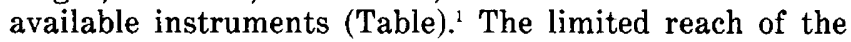
Ocutome should be taken into account preoperatively when planning vitrectomy in eyes with axial lengths substantially greater than $25 \mathrm{~mm}$. This will prevent loss of time and unexpected procedural or equipment changes intraoperatively when posterior vitreous removal is desired.

MATTHEW J. LIPMAN, MD

Charles A. Wilson, MD Cincinnati

This study was supported in part by an unrestricted grant from Research to Prevent Blindness Inc, New York, and from the Ohio Lions Eye Research Foundation, Columbus.

1. Klein RM, Katzin HM: Microsurgery of the Vitreous. Comparisons of Instrumentation, Techniques, and Philosophies. Baltimore, Williams \& Wilkins Co, 1978, pp 28-37.

\section{Treatment of Partly Accommodative Esotropia With a High Accommodative Convergence- Accommodation Ratio}

To the Editor.-In the June 1987 issue of the ARCHIVEs, the results were published of a study by Kushner et $\mathrm{al}^{1}$ on the treatment of accommodative convergence excess, with bilateral medial rectus recessions or recessions with 14$\mathrm{mm}$ posterior fixation sutures. Posterior fixation surgery was considered an unsatisfactory technique in this study, a conclusion based mainly on the frequent occurrence of overcorrections, although large undercorrections also occurred in the recession with posterior fixation suture group. Mims, ${ }^{2}$ in a letter to the editor in the September 1987 issue of the ARCHIVES, reached a similar conclusion: six overcorrections in a group of 30 patients.

We think that at least some of these overcorrections were caused by adding a large, uniform-dose posterior fixation operation to a recession that, by itself, was appropriate for the angle measured at distance fixation. It must be realized that posterior fixation surgery not only reduces the angle of squint measured at near fixation but also, albeit to a lesser extent, the angle measured at distance fixation. At first glance, it may seem that sewing the medial rectus to the globe slightly behind the equator should not result in a significant change of the position of the eye when the eye is in primary or in slightly adducted position.

We have found previously, however, in a computer model analysis, ${ }^{3}$ that the effect of the displacement of the medial rectus muscle by the posterior fixation suture is considerable and well explains the change of the angle measured at distance. Posterior fixation surgery on the medial recti should be performed in a tailored fashion, ie, bilateral 12-,
13-, and 14-mm posterior fixation of the medial recti will reduce a far esotropia of approximately $+4^{\circ},+6^{\circ}$, and $+8^{\circ}$, and a near esotropia of approximately $+10^{\circ},+13^{\circ}$, and $+16^{\circ}$, respectively, to $0 .^{4}$ At large doses $(\geq 14 \mathrm{~mm})$, the result becomes more unpredictable and depends progressively on axial length. ${ }^{3}$

\section{H. J. Simonsz, MD, PHD Freiburg West Germany}

1. Kushner BJ, Preslan MW, Morton GV: Treatment of partly accommodative esotropia with a high accommodative convergence-accommodation ratio. Arch Ophthalmol 1987;105:815-818.

2. Mims JL: Confirmation of Kushner's method for augmented recessions of the medial recti. Arch Ophthalmol 1987;105:1163.

3. Simonsz HJ, van Dijk B: Analysis of the dosage controversy in recess-resect and Faden surgery with the Robinson computer model of eye movements. Doc Ophthalmol 1988;67:237-252.

4. Kaufmann $\mathrm{H}$ : Wirkungsprinzipien und Resultate verschiedener Operationsmethoden bei Strabismus horizontalis. Ber Berufsverband Augenärzte Dtsch Arbeitskr Schielen, 1983, pp 30-39.

In Reply.-Any clinical trial of two treatment modalities only compares their relative efficacy when used in exactly the same manner in which they were used in that trial. Thus, it is appropriate for Dr Simonsz to question whether our poor results with the posterior fixation suture with recession operation resulted from our specific recession formula rather than the use of the posterior fixation suture per se. Our data, however, suggest that this is not the case.

Our retrospective review of patients with partly accommodative esotropia, with a high accommodative convergence-accommodation ratio, who were operated on between 1974 and 1980, indicated that in this group of patients the response to surgery (prism diopters per millimeter of recession) was inversely related to the accommodative convergence-accommodation ratio. I am unaware of any studies in the literature that contradict this finding. The data from our review suggest that the amount of recession that we utilized in our subsequent clinical trial, combined with the posterior fixation suture, would have been insufficient by itself to achieve adequate alignment in these patients; there would have been many undercorrections.

Dr Simonsz suggests that our large number of overcorrections in the posterior fixation suture group resulted from too generous a recession and possibly placing the posterior fixation suture too far from the limbus in some patients. $\mathrm{He}$ is undoubtedly correct that decreasing the size of the recession and shortening the distance from the limbus to the posterior fixation suture would have decreased the number of overcorrections. However, our posterior fixation group also had many undercorrections for near fixation. Nine $(43 \%)$ of 21 patients needed to remain in a bifocal lens postoperatively, which was significantly more than in our augmented recession group $(P<.05)$. Undoubtedly, decreasing the amount of recession, or shortening the distance between the limbus and the posterior fixation suture, would also have increased the number of undercorrections for near fixation. This supports the idea that the posterior fixation suture with recession operation is less predictable than a graded recession based on the distance deviation as well as the amount of convergence excess. There is no question that the graded recession operation is easier and safer to perform than the recession with posterior fixation suture operation.

Burton J. Kushner, MD Madison, Wis 